Article

\title{
Patient-Reported Expectations, Outcome and Satisfaction in Thoracic and Lumbar Spine Stabilization Surgery: A Prospective Study
}

\author{
Philipp Krauss, Clara Sonnleitner, Feline Reinartz, Bernhard Meyer@ and Hanno S. Meyer* \\ Department of Neurosurgery, Klinikum rechts der Isar, Technical University of Munich, Ismaninger Str. 22, \\ 81675 Munich, Germany; philipp.krauss@tum.de (P.K.); clarasonnleitner@yahoo.de (C.S.); feliner@web.de (F.R.); \\ Bernhard.Meyer@tum.de (B.M.) \\ * Correspondence: Hanno.Meyer@tum.de
}

Received: 27 September 2020; Accepted: 12 November 2020; Published: 16 November 2020

check for updates

\begin{abstract}
Patient-reported outcome measures (PROMs) have become an important aspect of quality control in modern healthcare. In this prospective observational study on 199 patients undergoing thoracolumbar stabilization surgery, we quantified preoperative expectations and PROMs at six and twelve months after surgery, and we investigated what constitutes patient satisfaction with the outcome. We used the visual analogue scale (VAS) for pain and the Oswestry Disability Index (ODI). Preoperative expectations were high (expected ODI: $9 \pm 13 \%$; leg pain: $1.0 \pm 1.4$; back pain: $1.3 \pm 1.5$ ). Pain and disability improved substantially, but expectations were mostly unrealistic (ODI expectation fulfilled after six months: $28 \%$ of patients; back pain: $48 \%$ ). However, satisfaction was high (70\% at six months after surgery). Satisfied patients had significantly better pain and disability outcomes and higher rates of expectation fulfillment than non-satisfied patients. Patients undergoing revision stabilization had worse outcomes than all other diagnosis groups. Prior stabilization surgery was identified as an independent risk factor for dissatisfaction. There were no preoperative pain or disability levels that predicted dissatisfaction. The data presented in this study can provide benchmarks for diagnosis-specific PROM targets in thoracolumbar stabilization surgery. Future studies should investigate whether satisfaction can be influenced, e.g., by discussing realistic outcome targets with patients ahead of surgery.
\end{abstract}

Keywords: lumbar spine; thoracic spine; spondylodesis; stabilization surgery; satisfaction; expectations; outcome; patient-reported outcome measures; visual analogue scale; Oswestry Disability Index

\section{Introduction}

In modern healthcare delivery models, such as value-based healthcare, care providers are rewarded based on patient health outcomes. Consequently, in orthopedic surgery and spinal surgery, patient satisfaction and other patient-reported outcome measures (PROMs) have come into focus not only as key indicators for quality assessment, but also as a potential basis for reimbursement. The National Health Service of the United Kingdom, for example, has been collecting PROMs for hip and knee replacement procedures [1].

To adequately assess individual PROMs in spinal surgery, realistic outcome targets have to be established for specific types of spinal disease and treatment. Moreover, one has to identify the factors that drive patient satisfaction first in order to improve it. It is unclear, for example, to what extent pain relief and disability improvement contribute to satisfaction after spinal surgery [2]. Psychological factors may play a role as well. In consumer psychology, satisfaction has been closely related to 
expectations according to expectation disconfirmation theory [3,4]. In fact, numerous studies have investigated the relationship between preoperative expectations and postoperative satisfaction in patients undergoing spine surgery [5-20]. Several found a positive and others found a negative association, and some have argued that the difference between expectations and actual outcome is key. A study on minimally invasive lumbar procedures concluded that the actual outcome itself, rather than the preoperative expectations or the expectation/outcome discrepancy, is the most important predictor of patient satisfaction [21].

The goal of this study was to quantify and investigate the relation of patient expectations and other patient factors on the one hand and PROMs regarding pain and disability as well as patient satisfaction on the other hand in a cohort of patients undergoing stabilization surgery of the thoracic and/or lumbar spine at a German academic level one spine center. While previous studies have mostly focused on degenerative spinal disease, we also included patients with spinal tumors, spondylodiscitis, and spinal fractures.

We found that pain and disability improve substantially after thoracic or lumbar stabilization surgery for various diseases. Even though the high preoperative patient expectations are often not fulfilled, postoperative satisfaction rates are high and remain high over time for most diagnosis groups. Fulfillment of expectations as well as good pain and disability outcomes are associated with satisfaction with the treatment result, but there are no preoperative pain, disability or expectation levels that predict satisfaction. Disability improvement and back pain reduction are important, but not the only drivers of patient satisfaction. Patients with degenerative disease undergoing revision stabilization surgery have worse pain and disability outcomes than all other diagnosis groups and a higher risk for dissatisfaction with the outcome.

\section{Materials and Methods}

This was a prospective observational single center study. All data were collected, encrypted, processed, and analyzed according to the study protocol approved by the local ethics committee (Technical University of Munich: 5749/13). All included patients gave their written consent.

Inclusion criteria for patient selection were patients aged 18 years or older and scheduled for thoracic or lumbar spine stabilization surgery at the Department of Neurosurgery at the Klinikum rechts der Isar, Technical University of Munich, Germany between the end of 2013 and 2015. Exclusion criteria were patients age less than 18 years; German language deficits or cognitive deficits/diseases preventing obtainment of informed consent to take part in the study; refusal to give written informed consent.

Stabilization surgery was indicated in patients with degenerative disease (including spinal stenosis and/or disk herniation with or without spondylolisthesis, spondylolysis, adjacent segment disease and/or implant failure after previous stabilization surgery), with spinal tumors (including masses compressing the spinal cord and/or nerve roots, and/or those with tumor-related fractures/instability), with spondylodiscitis, with traumatic fractures (including instable fractures and/or those with nerve root/spinal cord compression), and with osteoporotic fractures (including those with spinal cord and/or nerve root compression, and/or instability/kyphosis, and/or those at junctional zones). Stabilization surgery was performed either alone or in combination with decompression. Stabilization was either achieved by posterior pedicle screw-based instrumentation alone or in combination with an intercorporal fusion (transforaminal lumbar interbody fusion and/or extreme lateral interbody fusion and/or anterior lumbar interbody fusion)/vertebral body replacement (i.e., 360 degree fusion); cf. Table 1.

All patients underwent a standardized preoperative workup including pain assessment according to the visual analogue scale (VAS) for back and leg pain and the Oswestry Disability Index (ODI). In addition, patients were asked to specify, by means of these tools, the condition they expected to be at least satisfied with the outcome. At discharge, pain levels were evaluated again, and patients were asked whether they were satisfied with the outcome at this point (yes or no). Six and twelve months after surgery, pain levels, ODI and satisfaction with the outcome were evaluated again. Prior to 
spinal surgeries, the body mass index (BMI) and smoking status were documented for every patient at admission.

Table 1. Patient demographics according to type of stabilization surgery. Pts., patients; $\mathrm{f} / \mathrm{u}$, follow-up; disch., discharge; mo., months; BMI, body mass index. Age (years) and BMI $\left(\mathrm{kg} / \mathrm{m}^{2}\right)$ are given as mean \pm standard deviation. Note that 10 of the 91 patients that were excluded are not shown here because they eventually did not undergo stabilization surgery.

\begin{tabular}{ccccc}
\hline & \multicolumn{2}{c}{ Dorsal Instrumentation Only } & \multicolumn{2}{c}{ 360 Degree Fusion } \\
\hline Decompression & yes & no & yes & no \\
Pts. excluded & 26 & 7 & 43 & 5 \\
Pts. included (females) & $79(36)$ & $16(10)$ & $82(43)$ & $22(12)$ \\
Pts. w. f/u at disch./6/12/mo. & $79 / 64 / 54$ & $13 / 13 / 9$ & $79 / 64 / 59$ & $21 / 21 / 17$ \\
Females at disch./6/12/mo. & $36 / 30 / 27$ & $9 / 9 / 6$ & $41 / 35 / 31$ & $11 / 11 / 9$ \\
Age at day of inclusion & $67 \pm 13$ & $57 \pm 19$ & $66 \pm 13$ & $65 \pm 11$ \\
BMI at day of inclusion & $27 \pm 5$ & $26 \pm 4$ & $27 \pm 5$ & $28 \pm 5$ \\
\hline
\end{tabular}

Statistical analysis was performed using the software SPSS Statistics ${ }^{\mathrm{TM}}$ (version 25, IBM Corp, Armonk, New York, NY, USA). Normal distribution was confirmed according to the central limit theorem, the Kolmogorov-Smirnov and the Shapiro-Wilk tests. An unpaired two-tailed Student's $\mathrm{t}$ test was used to compare means between two groups (and corrected for alpha-error using the Holm-Bonferroni method). For ordinal variables, an unpaired Mann-Whitney $U$ test was used to compare two samples. For categorial values, the chi-square test was performed. Correlations were performed using Pearson's or Spearman's correlation, respectively. Factorial logistic regression was performed in selected cases. Data in text and graphs are shown as mean \pm standard deviation (SD), if not indicated otherwise. A $p$ value $\leq 0.05$ was considered statistically significant.

\section{Results}

\subsection{Study Population}

In total, 290 patients were screened for this study. Furthermore, 91 were excluded (64\% due to patient refusal, $22 \%$ due to German language deficits and $14 \%$ due to cognitive impairment). Consequently, 199 patients (101 female) were included (mean age: $65 \pm 13$ years; BMI: $27.3 \pm 5.1$; $17.6 \%$ were smokers). All underwent stabilization surgery (Table 1). Moreover, 119 had stabilization surgery for degenerative disease (34 of which had revision surgery after prior stabilization procedures, e.g., due to implant failure or adjacent segment disease), 29 had a spinal tumor, 29 had spondylodiscitis, 14 had traumatic, and eight had osteoporotic vertebral fractures. Follow-up was $96 \%$ at discharge, $81 \%$ after six months and $70 \%$ after twelve months. In 74 cases, patients had prior lumbar or thoracic spine surgery (41 of them prior stabilization surgery).

\subsection{Preoperative Symptom Burden}

Patients of all diagnosis groups had substantial back pain before surgery (overall average: $6.7 \pm 3.0$; Table 2) that was higher than leg pain $(4.7 \pm 3.7)$. Leg pain was more intense in patients with a degenerative primary diagnosis (5.9 \pm 3.2 vs. $2.8 \pm 3.4, p<0.001)$. Disability (ODI), on the other hand, was more severe in non-degenerative cases ( $53 \pm 25$ vs. $45 \pm 20, p<0.01$ ). Of note, this was not true for patients with a degenerative disease undergoing revision stabilization surgery, who had disability scores that were comparable to non-degenerative patients and much higher than those of patients with primary stabilization surgery ( $53 \pm 18$ vs. $42 \pm 20, p<0.01$ ). Female patients reported higher back pain prior to surgery $(7.3 \pm 2.9$ vs. $6.1 \pm 3.1 ; p<0.01)$. 
Table 2. Preoperative patient symptom burden, patient-reported expectations, and outcome by primary diagnosis over time (mean \pm standard deviation). VAS: visual analogue scale; ODI: Oswestry Disability Index. Expect.: expectation. Disch.: discharge. Exp. Fulf.: rate of patients with fulfilled expectations. Mo: months.

\begin{tabular}{|c|c|c|c|c|c|c|c|}
\hline & Preop. & Expect. & Disch. & $6 \mathrm{Mo.}$ & Exp. Fulf. & 12 Mo. & Exp. Fulf. \\
\hline \multicolumn{8}{|l|}{ Back pain (VAS) } \\
\hline All Patients & $6.7 \pm 3.0$ & $1.3 \pm 1.5$ & $4.8 \pm 3.0$ & $2.9 \pm 2.9$ & $48 \%$ & $2.6 \pm 3.1$ & $54 \%$ \\
\hline Degenerative & $6.8 \pm 2.7$ & $1.6 \pm 1.6$ & $4.8 \pm 3.2$ & $3.3 \pm 3.0$ & $43 \%$ & $3.0 \pm 3.2$ & $50 \%$ \\
\hline no revision & $6.9 \pm 2.5$ & $1.4 \pm 1.4$ & $4.9 \pm 3.1$ & $2.8 \pm 2.8$ & $46 \%$ & $2.6 \pm 3.1$ & $57 \%$ \\
\hline revision stabilization & $6.8 \pm 3.1$ & $1.9 \pm 1.9$ & $4.5 \pm 3.4$ & $4.6 \pm 3.3$ & $35 \%$ & $3.9 \pm 3.4$ & $33 \%$ \\
\hline Non-Degenerative & $6.4 \pm 3.5$ & $0.9 \pm 1.2$ & $4.8 \pm 2.8$ & $2.1 \pm 2.7$ & $56 \%$ & $1.8 \pm 2.6$ & $63 \%$ \\
\hline Infection & $6.9 \pm 3.3$ & $1.2 \pm 1.4$ & $4.2 \pm 2.8$ & $1.4 \pm 2.6$ & $68 \%$ & $1.6 \pm 2.7$ & $71 \%$ \\
\hline Tumor & $7.0 \pm 3.5$ & $0.9 \pm 1.3$ & $5.5 \pm 2.9$ & $2.8 \pm 2.5$ & $38 \%$ & $1.0 \pm 1.8$ & $73 \%$ \\
\hline Trauma & $5.4 \pm 3.7$ & $0.4 \pm 0.7$ & $5.4 \pm 1.6$ & $4.0 \pm 3.9$ & $29 \%$ & $2.0 \pm 2.3$ & $43 \%$ \\
\hline Osteoporosis & $4.8 \pm 3.5$ & $0.8 \pm 0.9$ & $4.2 \pm 2.9$ & $1.4 \pm 1.9$ & $69 \%$ & $2.8 \pm 3.1$ & $55 \%$ \\
\hline \multicolumn{8}{|l|}{ Leg pain (VAS) } \\
\hline All Patients & $4.7 \pm 3.7$ & $1.6 \pm 2.6$ & $1.6 \pm 2.6$ & $1.5 \pm 2.6$ & $73 \%$ & $1.3 \pm 2.5$ & $77 \%$ \\
\hline Degenerative & $5.9 \pm 3.2$ & $1.2 \pm 1.4$ & $2.2 \pm 2.9$ & $1.9 \pm 2.9$ & $66 \%$ & $1.7 \pm 2.8$ & $71 \%$ \\
\hline no revision & $6.2 \pm 3.1$ & $1.1 \pm 1.1$ & $2.2 \pm 2.9$ & $1.8_{ \pm} 2.7$ & $68 \%$ & $1.5 \pm 2.7$ & $75 \%$ \\
\hline revision stabilization & $5.4 \pm 3.6$ & $1.4 \pm 1.8$ & $2.1 \pm 2.9$ & $4.6 \pm 3.3$ & $63 \%$ & $2.2 \pm 3.1$ & $62 \%$ \\
\hline Non-Degenerative & $2.8 \pm 3.4$ & $0.7 \pm 1.2$ & $0.8 \pm 1.9$ & $0.7 \pm 1.8$ & $85 \%$ & $0.5 \pm 1.5$ & $89 \%$ \\
\hline Infection & $2.9 \pm 3.4$ & $0.8 \pm 1.0$ & $0.6 \pm 1.3$ & $0.1 \pm 0.4$ & $95 \%$ & $0.8 \pm 2.1$ & $82 \%$ \\
\hline Tumor & $2.9 \pm 3.8$ & $0.6 \pm 1.4$ & $0.5 \pm 1.8$ & $0.8 \pm 1.9$ & $85 \%$ & $0.2 \pm 0.6$ & $91 \%$ \\
\hline Trauma & $1.0 \pm 1.9$ & $0.3 \pm 0.5$ & $1.1 \pm 2.5$ & $1.7 \pm 2.9$ & $71 \%$ & $0.7 \pm 1.9$ & $86 \%$ \\
\hline Osteoporosis & $3.3 \pm 3.4$ & $0.9 \pm 1.4$ & $1.8 \pm 2.7$ & $1.2 \pm 2.2$ & $77 \%$ & $0.0 \pm 0.0$ & $100 \%$ \\
\hline \multicolumn{8}{|l|}{ ODI } \\
\hline All Patients & $48 \pm 22$ & $9 \pm 13$ & - & $28 \pm 25$ & $28 \%$ & $24 \pm 23$ & $36 \%$ \\
\hline Degenerative & $45 \pm 20$ & $10 \pm 13$ & - & $30 \pm 25$ & $22 \%$ & $27 \pm 24$ & $33 \%$ \\
\hline no revision & $42 \pm 20$ & $6 \pm 7$ & - & $23 \pm 23$ & $27 \%$ & $21 \pm 22$ & $38 \%$ \\
\hline revision stabilization & $53 \pm 18$ & $20 \pm 19$ & - & $46 \pm 25$ & $10 \%$ & $41 \pm 26$ & $19 \%$ \\
\hline Non-Degenerative & $53 \pm 25$ & $9 \pm 12$ & - & $23 \pm 23$ & $40 \%$ & $19 \pm 20$ & $41 \%$ \\
\hline Infection & $58 \pm 25$ & $12 \pm 15$ & - & $15 \pm 18$ & $50 \%$ & $12 \pm 18$ & $53 \%$ \\
\hline Tumor & $49 \pm 19$ & $7 \pm 8$ & - & $36 \pm 28$ & $31 \%$ & $23 \pm 22$ & $27 \%$ \\
\hline Trauma & $54 \pm 39$ & $1 \pm 3$ & - & $21 \pm 26$ & $29 \%$ & $19 \pm 22$ & $29 \%$ \\
\hline Osteoporosis & $49 \pm 25$ & $11 \pm 12$ & - & $23 \pm 23$ & $38 \%$ & $25 \pm 20$ & $45 \%$ \\
\hline
\end{tabular}


Table 2. Cont.

\begin{tabular}{|c|c|c|c|c|c|c|c|}
\hline & Preop. & Expect. & Disch. & $6 \mathrm{Mo.}$ & Exp. Fulf. & 12 Mo. & Exp. Fulf \\
\hline \multicolumn{8}{|l|}{ Satisfaction Rate } \\
\hline All Patients & - & - & $82 \%$ & $70 \%$ & & $78 \%$ & \\
\hline Degenerative & - & - & $79 \%$ & $65 \%$ & & $75 \%$ & \\
\hline no revision & - & - & $82 \%$ & $74 \%$ & & $84 \%$ & \\
\hline revision stabilization & - & - & $72 \%$ & $47 \%$ & & $54 \%$ & \\
\hline Non-Degenerative & - & - & $86 \%$ & $78 \%$ & & $85 \%$ & \\
\hline Infection & - & - & $93 \%$ & $92 \%$ & & $89 \%$ & \\
\hline Tumor & - & - & $82 \%$ & $71 \%$ & & $100 \%$ & \\
\hline Trauma & - & - & $88 \%$ & $57 \%$ & & $71 \%$ & \\
\hline Osteoporosis & & & $77 \%$ & $69 \%$ & & $75 \%$ & \\
\hline
\end{tabular}




\subsection{Postoperative Outcome for Pain and Disability}

The postoperative outcome was good with substantial improvement of back pain, leg pain and disability at six months after surgery compared to the preoperative status, irrespective of the primary diagnosis (Table 2, Figure 1). Pain and disability outcomes remained at least stable or improved between six and twelve months after surgery. Outcomes were diagnosis-specific. Compared with the preoperative status, patients with degenerative disease undergoing revision stabilization surgery still had higher disability $(p<0.001)$ after six months, but now they also had more back pain $(p=0.01)$ than patients with degenerative disease who had primary stabilization surgery. They also had higher disability $(p<0.001)$ and leg pain $(p<0.001)$ than patients with non-degenerative diagnoses. Patients with degenerative disease still had more leg pain than non-degenerative cases $(p<0.01)$, and female patients still had significantly higher back pain ( $3.4 \pm 3.0$ vs. $2.3 \pm 2.8 ; p=0.03)$. Leg pain or ODI scores did not differ between sexes.

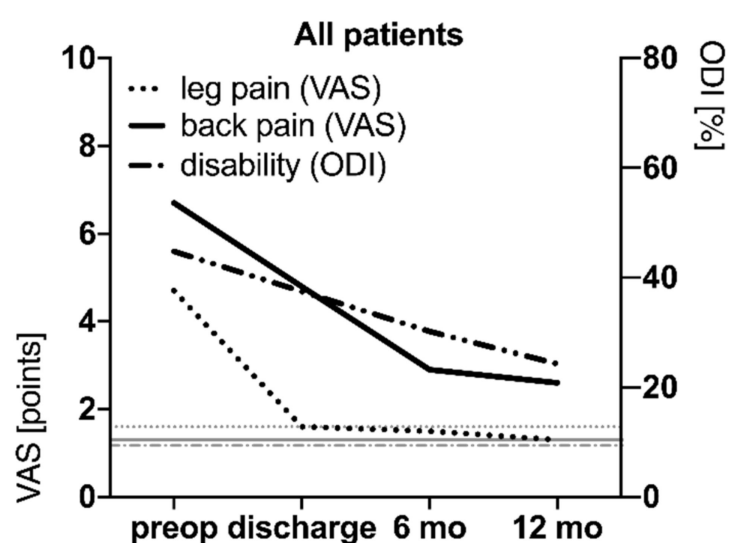

(a)

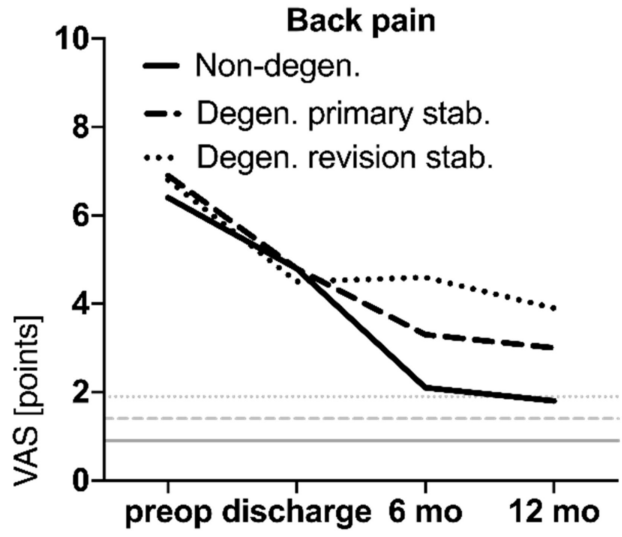

(c)

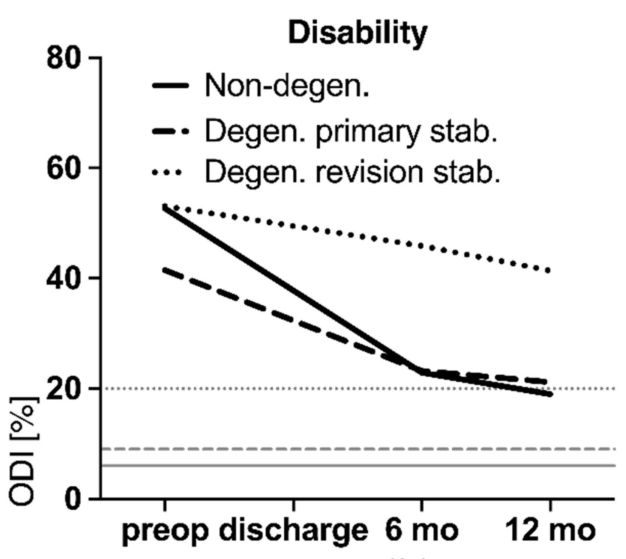

(b)

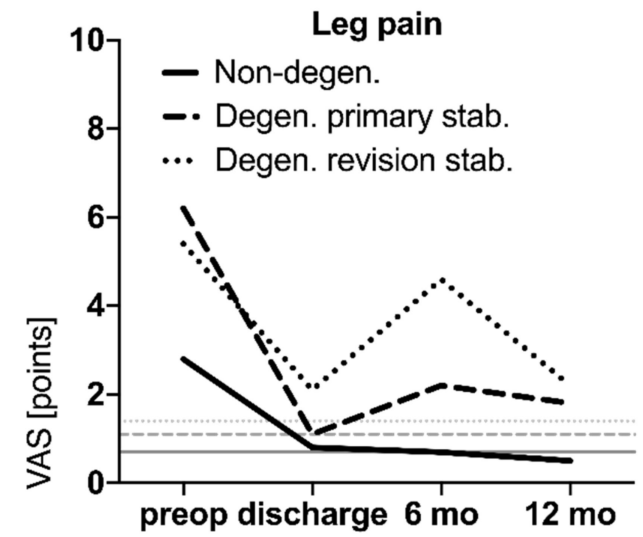

(d)

Figure 1. Pain and disability outcome (mean values) over time. (a) Back pain, leg pain and disability outcome of all patients. (b) Disability outcome for non-degenerative subgroup and subgroups with degenerative diagnosis (primary stabilization and revision stabilization), as indicated by different graph styles. (c), (d) Same as (b), but for back pain and leg pain. Expectations are indicated by gray lines. VAS: visual analogue scale; ODI: Oswestry Disability Index.

\subsection{Patient Expectations}

Patients with all diagnoses had high expectations that were, on average, not fulfilled (Table 2, Figure 1). Patients with degenerative disease had slightly lower expectations for back $(p<0.01)$ and leg $(p<0.01)$ pain outcomes than patients with non-degenerative diagnoses (i.e., they expected higher 
pain levels). This was mostly due to patients undergoing revision stabilization surgery, who had lower pain expectations than those with primary stabilization for degenerative disease. Expectations for disability outcomes were much lower in the revision stabilization group than in the primary stabilization $(p<0.001)$ and all other groups. We measured the realism of preoperative expectations as the rate of patients who had their expectations fulfilled at six months after surgery. Expectations were more realistic for leg pain ( $73 \%$ of all patients had their expectations fulfilled six months after surgery) than for back pain (48\%). The expectations for disability improvement were least realistic (28\%).

The realism of expectations was diagnosis-specific. Patients with degenerative disease had less realistic expectations for leg pain (66\% had their expectations fulfilled), back pain (43\%) and disability outcome (22\%) than patients with non-degenerative diagnoses $(85 \%, 56 \%$ and $40 \%$, respectively). This was especially true for patients undergoing revision stabilization surgery, who had the least realistic expectations for disability (10\%) and leg pain (63\%) outcome of all diagnosis groups and, together with trauma patients, the least realistic expectations for back pain outcome (trauma: 29\%; revision stabilization: $35 \%$ ). The difference in realism of expectations was statistically significant for disability expectations ( $p=0.03$, chi-square test).

\subsection{Patient Satisfaction}

Despite low rates of expectation fulfillment, the rate of patients satisfied with their outcome was high at all points in time (Table 2, Figure 1). It was highest at discharge with $81.8 \%$. Similar to pain and disability outcomes, satisfaction improved between six and twelve months $(69.8 \%$ vs. $78.4 \%$ ). Even assuming that all patients lost to follow-up were dissatisfied at twelve months yields that satisfaction remained at least stable over time $(69.8 \%$ vs. $67.3 \%)$. Patient satisfaction rates at six months were diagnosis-specific as well ( $p=0.01$, chi-square test). Patients with non-degenerative diseases were more likely to be satisfied ( $78 \%$ vs. $65 \%$ ), which was due to the group of patients with degenerative disease undergoing revision stabilization surgery, which had the lowest satisfaction rate of all $(47 \%)$. Satisfaction rates dropped between discharge and six months after surgery in all diagnosis groups.

\subsection{The Relation of Patient Factors and Outcome}

The rate of dissatisfied patients was higher in patients who had prior spine surgery (Table 3) in accordance with the worse outcome of patients undergoing revision stabilization surgery (see above). Among females, there were also more dissatisfied patients. Smoking status and BMI did not make a difference regarding satisfaction.

A factorial logistic regression model of patient factors identified prior stabilization surgery $(p<0.001,12$ months) and female gender ( $p=0.01,6$ months) as significant independent risk factors for dissatisfaction.

\subsection{The Relation of Preoperative Status, Expectations, Pain and Disability Outcome and Satisfaction}

Patients that were satisfied after six months hardly differed from non-satisfied patients with regard to preoperative pain and disability levels and expectations (Table 4). In patients with degenerative disease, preoperative ODI levels were higher and absolute back pain expectation levels were lower (i.e., they expected a higher VAS level) than in non-satisfied patients. The expected absolute and relative improvement of back pain, leg pain and disability levels also did not differ between satisfied and non-satisfied patients, and only in patients with degenerative disease was there a weak negative correlation between expectations for disability improvement (ODI reduction) and satisfaction $(\mathrm{r}=-0.2$, $p=0.04)$.

In contrast, all outcome measures were significantly better in satisfied patients of all major diagnosis groups, except for leg pain in patients undergoing revision stabilization surgery. Moreover, the percentages of patients with fulfilled back pain and disability expectations were significantly higher in satisfied compared to dissatisfied patients in all major diagnosis groups. This was also the case for fulfilled leg pain expectations in non-degenerative patients. 
Table 3. Percentage of patients dissatisfied with the outcome at six months according to patient factors. BMI: body mass index; $p$ values are based on a chi-square test; bold text indicates significant differences $(p \leq 0.05)$. Deg.: Degenerative; rev.: revision.

\begin{tabular}{|c|c|c|c|c|c|c|c|c|c|c|}
\hline & \multicolumn{2}{|c|}{ Sex } & \multicolumn{2}{|c|}{ Smoker } & \multicolumn{4}{|c|}{ BMI $\left(\mathrm{kg} / \mathrm{m}^{2}\right)$} & \multicolumn{2}{|c|}{ Prior Spinal Surgery } \\
\hline & Male & Female & Yes & No & $<25$ & $25<30$ & $30<35$ & $\geq 35$ & Yes & No \\
\hline \multirow[t]{2}{*}{ All patients } & $22.1 \%$ & $37.6 \%$ & $39.3 \%$ & $28.4 \%$ & $23.6 \%$ & $35.7 \%$ & $30.3 \%$ & $33.3 \%$ & $42.2 \%$ & $22.4 \%$ \\
\hline & \multicolumn{2}{|c|}{$p=0.04$} & \multicolumn{2}{|c|}{$p=0.26$} & \multicolumn{4}{|c|}{$p=0.58$} & \multicolumn{2}{|c|}{$p<0.01$} \\
\hline \multirow[t]{2}{*}{ Degenerative } & $25.0 \%$ & $42.9 \%$ & 40.9 & 32.9 & $24.1 \%$ & $40.1 \%$ & $36.0 \%$ & $27.3 \%$ & $44.4 \%$ & $24.0 \%$ \\
\hline & \multicolumn{2}{|c|}{$p=0.06$} & \multicolumn{2}{|c|}{$p=0.49$} & \multicolumn{4}{|c|}{$p=0.53$} & \multicolumn{2}{|c|}{$p=0.03$} \\
\hline \multirow[t]{2}{*}{ Deg. no rev. } & $18.8 \%$ & $32.5 \%$ & $18.2 \%$ & $27.9 \%$ & $14.3 \%$ & $26.2 \%$ & $35.3 \%$ & $28.6 \%$ & $31.8 \%$ & $24.0 \%$ \\
\hline & \multicolumn{2}{|c|}{$p=0.19$} & \multicolumn{2}{|c|}{$p=0.50$} & \multicolumn{4}{|c|}{$p=0.51$} & \multicolumn{2}{|c|}{$p=0.49$} \\
\hline \multirow[t]{2}{*}{ Deg. revision } & $37.5 \%$ & $68.8 \%$ & $63.6 \%$ & $47.6 \%$ & $50.0 \%$ & $72.7 \%$ & $37.5 \%$ & $25.0 \%$ & - & - \\
\hline & \multicolumn{2}{|c|}{$p=0.08$} & \multicolumn{2}{|c|}{$p=0.39$} & \multicolumn{4}{|c|}{$p=0.29$} & \multicolumn{2}{|c|}{-} \\
\hline \multirow[t]{2}{*}{ Non-deg. } & $17.2 \%$ & $27.6 \%$ & $21.2 \%$ & $33.3 \%$ & $24.0 \%$ & $26.3 \%$ & $0.0 \%$ & $50.0 \%$ & $30.0 \%$ & $20.8 \%$ \\
\hline & \multicolumn{2}{|c|}{$p=0.35$} & \multicolumn{2}{|c|}{$p=0.49$} & \multicolumn{4}{|c|}{$p=0.29$} & \multicolumn{2}{|c|}{$p=0.53$} \\
\hline
\end{tabular}


Table 4. Patient-reported preoperative symptom burden, expectations and pain and disability outcomes (mean \pm standard deviation) in satisfied and non-satisfied patients (6 month outcomes). Percentages indicate rates of patients who had their expectation fulfilled. VAS: visual analogue scale; ODI: Oswestry Disability Index; sat.: satisfied; Deg.: Degenerative. A $p$ value $\leq 0.05$ was considered significant.

\begin{tabular}{|c|c|c|c|c|c|c|c|c|c|}
\hline \multirow[b]{2}{*}{ All Patients } & \multicolumn{3}{|c|}{ Preoperative } & \multicolumn{3}{|c|}{ Expectations } & \multicolumn{3}{|c|}{6 Months } \\
\hline & Sat. & Non-Sat. & $p$ & Sat. & Non-Sat. & $p$ & Sat. & Non-Sat. & $p$ \\
\hline Back pain [VAS] & $6.5 \pm 3.1$ & $6.5 \pm 2.9$ & 0.94 & $1.1 \pm 1.3$ & $1.7 \pm 1.6$ & 0.02 & $2.0 \pm 2.5(58 \%)$ & $5.0 \pm 2.9(22 \%)$ & $<0.001(<0.001)$ \\
\hline Leg pain [VAS] & $4.5 \pm 3.8$ & $5.0 \pm 3.5$ & 0.40 & $0.9 \pm 1.4$ & $1.2 \pm 1.5$ & 0.17 & $0.9 \pm 2.0(80 \%)$ & $2.7 \pm 3.3(57 \%)$ & $<0.001(<0.01)$ \\
\hline Disability [ODI] & $45 \pm 23$ & $50 \pm 22$ & 0.23 & $9 \pm 13$ & $10 \pm 12$ & 0.17 & $18 \pm 20(39 \%)$ & $50 \pm 22(2 \%)$ & $<0.001(<0.001)$ \\
\hline \multicolumn{10}{|l|}{ Degenerative } \\
\hline Back pain [VAS] & $6.7 \pm 2.9$ & $6.9 \pm 2.7$ & 0.77 & $1.3 \pm 1.5$ & $1.9 \pm 1.7$ & 0.04 & $2.3 \pm 2.6(54 \%)$ & $5.5 \pm 2.7(21 \%)$ & $<0.001(<0.01)$ \\
\hline Leg pain [VAS] & $6.0 \pm 3.4$ & $5.7 \pm 3.3$ & 0.63 & $1.1 \pm 1.4$ & $1.3 \pm 1.5$ & 0.59 & $1.4 \pm 2.4(72 \%)$ & $2.9 \pm 3.4(57 \%)$ & $0.03(0.14)$ \\
\hline Disability [ODI] & $42 \pm 21$ & $50 \pm 18$ & 0.04 & $9 \pm 14$ & $10 \pm 12$ & 0.26 & $18.2 \pm 19.8(33 \%)$ & $52.7 \pm 18.6(0 \%)$ & $<0.001(<0.001)$ \\
\hline \multicolumn{10}{|l|}{ Deg. No Revision } \\
\hline Back pain [VAS] & $6.7 \pm 2.7$ & $6.8 \pm 2.6$ & 0.94 & $1.2 \pm 1.4$ & $1.7 \pm 1.4$ & 0.18 & $2.1 \pm 2.4(54 \%)$ & $4.8 \pm 2.8(22 \%)$ & $<0.001(0.02)$ \\
\hline Leg pain [VAS] & $5.9 \pm 3.4$ & $6.6 \pm 2.7$ & 0.43 & $0.9 \pm 1.1$ & $1.5 \pm 1.5$ & 0.12 & $1.2 \pm 2.0(73 \%)$ & $3.2 \pm 3.6(56 \%)$ & $0.03(0.17)$ \\
\hline Disability [ODI] & $39 \pm 21$ & $48 \pm 19$ & 0.09 & $6 \pm 7$ & $4 \pm 4$ & 0.95 & $13.3 \pm 14.1(37 \%)$ & $49.4 \pm 19.8(0 \%)$ & $<0.001(<0.01)$ \\
\hline \multicolumn{10}{|l|}{ Deg. Revision } \\
\hline Back pain [VAS] & $6.5 \pm 3.6$ & $6.9 \pm 2.8$ & 0.72 & $1.7 \pm 1.9$ & $2.3 \pm 2.0$ & .043 & $2.7 \pm 3.2(53 \%)$ & $6.3 \pm 2.4(19 \%)$ & $<0.01(0.04)$ \\
\hline Leg pain [VAS] & $6.2 \pm 3.7$ & $4.6 \pm 3.6$ & 0.22 & $1.9 \pm 2.0$ & $1.0 \pm 1.5$ & 0.18 & $2.2 \pm 3.3(67 \%)$ & $2.5 \pm 3.4(59 \%)$ & $0.78(0.65)$ \\
\hline Disability [ODI] & $53 \pm 19$ & $52 \pm 17$ & 0.88 & $23 \pm 23$ & $16 \pm 14$ & 0.66 & $34.8 \pm 26.9(20 \%)$ & $56.5 \pm 17.1(0 \%)$ & $0.02(0.06)$ \\
\hline \multicolumn{10}{|l|}{ Non-Deg. } \\
\hline Back pain [VAS] & $6.3 \pm 3.5$ & $5.5 \pm 3.5$ & 0.47 & $0.8 \pm 1.0$ & $0.9 \pm 0.9$ & 0.61 & $1.6 \pm 2.5(65 \%)$ & $3.6 \pm 3.1(25 \%)$ & $0.03(0.01)$ \\
\hline Leg pain [VAS] & $2.2 \pm 3.1$ & $3.3 \pm 3.4$ & 0.31 & $0.6 \pm 1.2$ & $1.2 \pm 1.4$ & 0.16 & $0.3 \pm 1.0(93 \%)$ & $2.3 \pm 2.9(58 \%)$ & $0.04(<0.01)$ \\
\hline Disability [ODI] & $50 \pm 25$ & $49 \pm 31$ & 0.89 & $7 \pm 11$ & $10 \pm 12$ & 0.49 & $17.9 \pm 19.9(49 \%)$ & $40.5 \pm 27.1(8 \%)$ & $<0.01(0.01)$ \\
\hline
\end{tabular}


However, fulfillment of expectations was not required to report satisfaction with the outcome, especially regarding disability (61\% of satisfied patients did not have their ODI expectation fulfilled) and back pain ( $41 \%$ of satisfied patients had missed their expectation). Fulfillment of disability expectations made satisfaction extremely likely (only $2 \%$ of non-satisfied patients had their ODI expectations fulfilled). Factorial logistic regression analysis revealed that meeting expectations for disability $(p<0.01)$ had the highest impact and for back pain $(p=0.03)$ the second highest impact on satisfaction.

Higher expectations correlated significantly with the outcome for pain and disability outcome measures at 6 months (Figure 2), i.e., patients expecting a better outcome were more likely to achieve a better outcome.

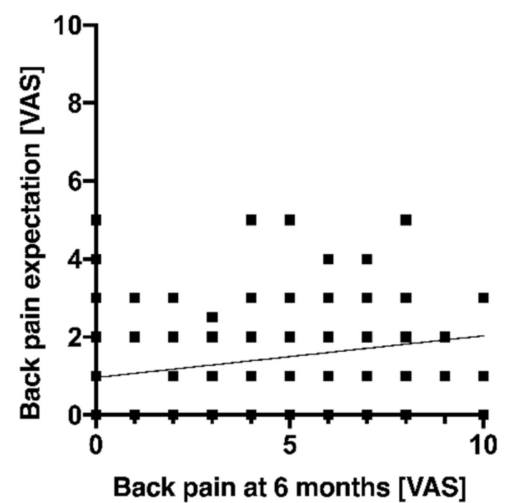

(a)

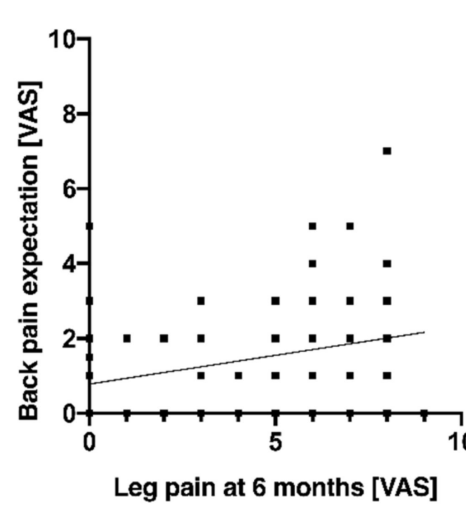

(b)

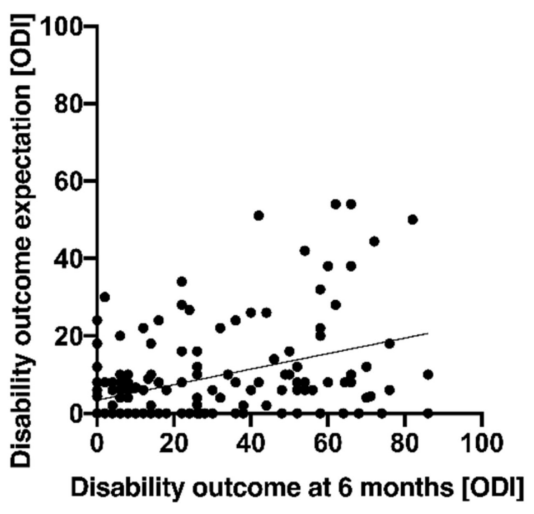

(c)

Figure 2. Preoperative patient expectations vs. outcomes after 6 months. (a) Back pain (VAS; Pearson's correlation: $r=0.22, p<0.01$ ). (b) Leg pain (VAS; Pearson's correlation: $r=0.29, p<0.001$ ). (c) Disability (ODI; Spearman's rho: $r=0.39, p<0.001$ ). Linear regression lines are plotted for visualization. VAS: visual analogue scale; ODI: Oswestry Disability Index.

To further investigate whether preoperative symptom burden can predict dissatisfaction, a receiver operating characteristic analysis of satisfaction vs. preoperative back pain (VAS), leg pain (VAS) and disability (ODI) was performed. This did not reveal a viable cut-off value to predict satisfaction with an area under the curve (AUC) of 0.516, 0.500 and 0.515, respectively. Accordingly, patients who reported maximum levels (10 points on the VAS) of back pain ( $\mathrm{n}=40$ patients) or leg pain ( $\mathrm{n}=18$ patients) prior to surgery did not have a significantly different rate of expectation fulfillment (back pain: $p=0.49$; leg pain: $p=0.96$ ) nor were they less satisfied with treatment after 6 months (back pain: $p=0.07$; leg pain: $p=0.29$ ) and 12 months (back pain: $p=0.83$; leg pain: $p=0.48$ ).

We also analyzed the relation of expectation-actuality differences and satisfaction. Smaller absolute expectation-actuality differences and higher degrees of expectation fulfillment were associated with satisfaction. The correlations were strongest for disability $(\mathrm{r}=-0.58, p<0.001$ for absolute differences and $\mathrm{r}=0.56, p<0.001$ for degree of fulfillment; Spearman's rho) and back pain ( $\mathrm{r}=-0.36$, $p<0.001 / \mathrm{r}=0.41, p<0.001)$. For leg pain, there were only weak correlations $(\mathrm{r}=-0.18, p=0.03 / \mathrm{r}=0.20$, $p=0.01)$.

\section{Discussion}

Facing a paradigm shift towards modern health care delivery models such as value-based care that put the patient's condition (rather than the specialty or the intervention) into focus [22-24], patient-reported outcomes have become popular. It has been suggested that health outcomes should cover the full cycle of care and track the patient's health status after care is completed [25]. In spinal surgery, specific PROMs (e.g., of pain, such as the VAS, and of disability, such as the ODI), as well as more general PROMs such as patient satisfaction with the outcome, have been the subject of numerous studies $[2,5-20,26,27]$. We prospectively investigated patient-reported pain and disability 
levels and satisfaction in patients undergoing stabilization surgery of the thoracic and/or lumbar spine for degenerative disease, tumors, infection and traumatic or osteoporotic fractures. We found that for most patients and most diagnoses, outcomes are good with substantial improvement of pain and disability and high satisfaction rates. We also found that outcomes are disease-specific: patients undergoing revision stabilization surgery, for example, have significantly worse outcomes than those with degenerative disease that are scheduled for primary stabilization. Our data can serve as benchmarks once patient-reported outcomes are actually intended to be used as indicators for quality control and potentially as a basis for reimbursement of spine surgery providers.

The outcomes we observed remained stable between six and twelve months after surgery for all diagnosis groups, also when correcting for patients lost to follow-up. This suggests that six-month follow-up can replace twelve-month follow-up in future studies on outcomes in thoracolumbar spinal stabilization surgery. Similar findings were recently reported comparing twelve and 24 month follow-ups in spinal surgery [28].

In our study, female patients presented with higher levels of back pain throughout all points in time. However, relative back pain improvements over time were similar to males. This is in accordance with prior reports on sex differences in spinal surgery, suggesting no gender-specific difference in outcome after spinal surgery [29,30].

Patient satisfaction with the outcome has always been a focus of surgeons treating diseases causing pain, such as spinal surgeons. Patient satisfaction is linked to quality of life and could be influenced by various factors not directly linked to therapy, such as BMI, sex, smoking habits and age $[7,31-33]$. Psychiatric diseases, such as anxiety and depression, have been shown to be related to the fulfillment of expectations and patient satisfaction as well [34,35]. Moreover, patient expectations have been proposed to influence satisfaction analogous to consumer psychology [3,4]. Identifying patients most likely to be satisfied and predicting the risk of dissatisfaction with the treatment outcome have become major concerns in spinal surgery, but results of studies have been contradictory [36-38]. In the present study, satisfaction with treatment was high at discharge, six months, and twelve months after thoracic/lumbar stabilization surgery $(82 \%, 70 \%$ and $78 \%$, respectively). It is possible that the drop between discharge and six months is based on the difference between satisfaction with treatment and satisfaction with outcome. At discharge, patients may be influenced by positive experiences they had during the hospital stay, and they cannot assess whether they actually benefitted from surgery in regard to regular activities or quality of life yet.

Understanding what drives patient satisfaction potentially enables surgeons to improve the outcome. Our study suggests, as expected, that the actual pain and disability outcome is of paramount importance for patient satisfaction, in accordance with a previous study on expectations and satisfaction in minimally invasive lumbar fusion surgery [21]. Moreover, satisfied patients had much better pain and disability outcomes, and fulfillment of disability expectations and back pain expectations were strongly associated with satisfaction. However, there is more to satisfaction than fulfillment of these expectations alone, as the majority of satisfied patients did not have their disability expectation fulfilled and almost half did not have their back pain expectation fulfilled. Maybe patients learn to adapt their expectations to the actual outcome, and most likely, other outcomes and patient factors that were not measured in this study play a role in inducing satisfaction as well (e.g., health-related quality of life, or psychiatric diseases).

Most of the preoperatively identifiable patient properties that we investigated, such as the symptom burden and the expectations, did not predict satisfaction in thoracolumbar stabilization surgery: expected pain and disability levels and absolute or relative expected improvement did not differ between satisfied and unsatisfied patients, and neither did preoperative pain and disability levels differ. The former is again in accordance with the study mentioned above [21]. Of note, not even patients reporting extreme preoperative pain levels (VAS 10) were less likely to be satisfied. Merely, in patients with degenerative disease, we found that those who had higher expectations for disability improvement were more likely to be dissatisfied. However, this was a weak correlation. Body mass 
index did not play a role, and neither did smoking status. Female patients were actually less satisfied, but the most eminent patient-specific factor that was associated with low satisfaction rates was the diagnosis. The subgroup of patients undergoing revision stabilization surgery, e.g., due to adjacent segment disease or due to implant failure, had the lowest satisfaction rate (and the worst pain and disability outcome) of all, and prior stabilization surgery was an independent risk factor for dissatisfaction. This raises the question of whether this finding should affect treatment decisions. When pain and disability are the main factors underlying the indication for surgery, the surgeon should discuss the lower chances for a good outcome with the patient. If the patient chooses to opt for surgery anyway, she/he might benefit from this as the expectations may be better adapted to the outcome, and our findings suggest that lower expectation-actuality differences increase the chance for satisfaction. On the other hand, one could also induce dissatisfaction when lowering the patient's expectations by promoting a self-fulfilling prophecy [39-42]. Future studies should investigate whether adjusting patient expectations according to established outcome targets can improve postoperative satisfaction. Regardless, there will remain many cases when the indication for surgery is based on other aspects that remain critical irrespective of pain and disability, such as the prevention of progressing spinal deformity and/or neurologic deficits. This is often true in non-degenerative disease, challenging the relevance of pain and disability outcomes and patient satisfaction in these patient groups. However, even in these patients, it is important to know what they expect and what their actual subjective outcome is, and one should aim at maximizing their satisfaction with the treatment as well.

\section{Conclusions}

Our data on patient expectations and patient-reported pain and disability outcomes as well as satisfaction in thoracolumbar spinal surgery can serve as benchmark facing the implementation of value-based health care systems. Patients have unrealistic expectations, but pain and disability outcomes are good and patient satisfaction is high for most diagnoses. Preoperative symptom burden and expectations do not predict satisfaction, but similar to pain and disability outcomes, satisfaction rates depend on the diagnosis. Patients undergoing revision stabilization surgery have significantly worse pain and disability outcomes and are less satisfied than those with degenerative disease that are scheduled for primary stabilization and patients with non-degenerative diseases. Disability improvement and back pain reduction are important, but they are not the only drivers of patient satisfaction.

Author Contributions: Conceptualization, H.S.M. and P.K.; methodology, H.S.M. and P.K.; formal analysis, P.K., C.S. and H.S.M.; data curation, C.S. and F.R.; writing-original draft preparation, P.K. and H.S.M.; writing-review and editing, H.S.M. and P.K.; supervision, H.S.M. and B.M. All authors have read and agreed to the published version of the manuscript.

Funding: This research received no external funding.

Acknowledgments: The authors would like to thank Martin Vazan for fruitful discussions and Florian Ringel for his support.

Conflicts of Interest: The authors declare no conflict of interest.

\section{References}

1. Gentry, S.; Badrinath, P. Defining Health in the Era of Value-based Care: Lessons from England of Relevance to Other Health Systems. Cureus 2017, 9, e1079. [CrossRef]

2. Menendez, J.Y.; Omar, N.B.; Chagoya, G.; Tabibian, B.E.; Elsayed, G.A.; Walters, B.C.; Guthrie, B.L.; Hadley, M.N. Patient Satisfaction in Spine Surgery: A Systematic Review of the Literature. Asian Spine J. 2019, 13, 1047-1057. [CrossRef] [PubMed]

3. Oliver, R.L. Effect of Expectation and Disconfirmation on Postexposure Product Evaluations-an Alternative Interpretation. J. Appl. Psychol. 1977, 62, 480-486. [CrossRef]

4. Oliver, R.L. A Cognitive Model of the Antecedents and Consequences of Satisfaction Decisions. J. Mark. Res. 1980, 17, 460-469. [CrossRef] 
5. Abbott, A.D.; Tyni-Lenne, R.; Hedlund, R. Leg pain and psychological variables predict outcome 2-3 years after lumbar fusion surgery. Eur. Spine J. 2011, 20, 1626-1634. [CrossRef]

6. Carr, F.A.; Healy, K.M.; Villavicencio, A.T.; Nelson, E.L.; Mason, A.; Burneikiene, S.; Hernandez, T.D. Effect on clinical outcomes of patient pain expectancies and preoperative Mental Component Summary scores from the 36-Item Short Form Health Survey following anterior cervical discectomy and fusion. J. Neurosurg. Spine 2011, 15, 486-490. [CrossRef]

7. Cobo Soriano, J.; Sendino Revuelta, M.; Fabregate Fuente, M.; Cimarra Diaz, I.; Martinez Urena, P.; Deglane Meneses, R. Predictors of outcome after decompressive lumbar surgery and instrumented posterolateral fusion. Eur. Spine J. 2010, 19, 1841-1848. [CrossRef]

8. Culliton, S.E.; Bryant, D.M.; Overend, T.J.; MacDonald, S.J.; Chesworth, B.M. The relationship between expectations and satisfaction in patients undergoing primary total knee arthroplasty. J. Arthroplast. 2012, 27, 490-492. [CrossRef]

9. De Groot, K.I.; Boeke, S.; Passchier, J. Preoperative expectations of pain and recovery in relation to postoperative disappointment in patients undergoing lumbar surgery. Med. Care 1999, 37, 149-156. [CrossRef]

10. Den Boer, J.J.; Oostendorp, R.A.; Beems, T.; Munneke, M.; Evers, A.W. Continued disability and pain after lumbar disc surgery: The role of cognitive-behavioral factors. Pain 2006, 123, 45-52. [CrossRef]

11. Gepstein, R.; Arinzon, Z.; Adunsky, A.; Folman, Y. Decompression surgery for lumbar spinal stenosis in the elderly: Preoperative expectations and postoperative satisfaction. Spinal Cord 2006, 44, 427-431. [CrossRef] [PubMed]

12. Iversen, M.D.; Daltroy, L.H.; Fossel, A.H.; Katz, J.N. The prognostic importance of patient pre-operative expectations of surgery for lumbar spinal stenosis. Patient Educ. Couns. 1998, 34, 169-178. [CrossRef]

13. Lutz, G.K.; Butzlaff, M.E.; Atlas, S.J.; Keller, R.B.; Singer, D.E.; Deyo, R.A. The relation between expectations and outcomes in surgery for sciatica. J. Gen. Intern. Med. 1999, 14, 740-744. [CrossRef] [PubMed]

14. McGregor, A.H.; Dore, C.J.; Morris, T.P. An exploration of patients' expectation of and satisfaction with surgical outcome. Eur. Spine J. 2013, 22, 2836-2844. [CrossRef] [PubMed]

15. Ronnberg, K.; Lind, B.; Zoega, B.; Halldin, K.; Gellerstedt, M.; Brisby, H. Patients' satisfaction with provided care/information and expectations on clinical outcome after lumbar disc herniation surgery. Spine (Phila Pa 1976) 2007, 32, 256-261. [CrossRef]

16. Saban, K.L.; Penckofer, S.M. Patient expectations of quality of life following lumbar spinal surgery. J. Neurosci. Nurs. 2007, 39, 180-189. [CrossRef]

17. Soroceanu, A.; Ching, A.; Abdu, W.; McGuire, K. Relationship between preoperative expectations, satisfaction, and functional outcomes in patients undergoing lumbar and cervical spine surgery: A multicenter study. Spine (Phila Pa 1976) 2012, 37, E103-E108. [CrossRef]

18. Toyone, T.; Tanaka, T.; Kato, D.; Kaneyama, R.; Otsuka, M. Patients' expectations and satisfaction in lumbar spine surgery. Spine (Phila Pa 1976) 2005, 30, 2689-2694. [CrossRef]

19. Xiong, D.D.; Ye, W.; Xiao, R.; Miller, J.A.; Mroz, T.E.; Steinmetz, M.P.; Nagel, S.J.; Machado, A.G. Patient-reported allergies predict postoperative outcomes and psychosomatic markers after spine surgery. Spine J. 2019, 19, 121-130. [CrossRef]

20. Yee, A.; Adjei, N.; Do, J.; Ford, M.; Finkelstein, J. Do patient expectations of spinal surgery relate to functional outcome? Clin. Orthop. Relat. Res. 2008, 466, 1154-1161. [CrossRef]

21. Yoo, J.S.; Patel, D.V.; Mayo, B.C.; Massel, D.H.; Karmarkar, S.S.; Lamoutte, E.H.; Singh, K. Postoperative satisfaction following lumbar spinal fusion surgery: Patient expectation versus actuality. J. Neurosurg. Spine 2019, 31, 676-682. [CrossRef] [PubMed]

22. Porter, M.E.; Lee, T.H. The strategy that will fix health care. Harv. Bus. Rev. 2013, 91, 24.

23. Porter, M.E. A Strategy for Health Care Reform-Toward a Value-Based System. N. Engl. J. Med. 2009, 361, 109-112. [CrossRef] [PubMed]

24. Porter, M.E.; Guth, C. Redefining German Health Care; Springer Press: Berlin/Heidelberg, Germany, 2012.

25. Porter, M.E. Measuring Health Outcomes: The Outcome Hierarchy. N. Engl. J. Med. 2010, 363, $2477-2481$. [CrossRef]

26. Stokes, O.M.; Cole, A.A.; Breakwell, L.M.; Lloyd, A.J.; Leonard, C.M.; Grevitt, M. Do we have the right PROMs for measuring outcomes in lumbar spinal surgery? Eur. Spine J. 2017, 26, 816-824. [CrossRef] 
27. Falavigna, A.; Dozza, D.C.; Teles, A.R.; Wong, C.C.; Barbagallo, G.; Brodke, D.; Al-Mutair, A.; Ghogawala, Z.; Riew, K.D. Current Status of Worldwide Use of Patient-Reported Outcome Measures (PROMs) in Spine Care. World Neurosurg. 2017, 108, 328-335. [CrossRef]

28. Staartjes, V.E.; Siccoli, A.; De Wispelaere, M.P.; Schroder, M.L. Patient-reported outcomes unbiased by length of follow-up after lumbar degenerative spine surgery: Do we need 2 years of follow-up? Spine J. 2019, 19, 637-644. [CrossRef]

29. Siccoli, A.; Staartjes, V.E.; De Wispelaere, M.P.; Schroder, M.L. Gender differences in degenerative spine surgery: Do female patients really fare worse? Eur. Spine J. 2018, 27, 2427-2435. [CrossRef]

30. Triebel, J.; Snellman, G.; Sanden, B.; Stromqvist, F.; Robinson, Y. Women do not fare worse than men after lumbar fusion surgery: Two-year follow-up results from 4780 prospectively collected patients in the Swedish National Spine Register with lumbar degenerative disc disease and chronic low back pain. Spine J. 2017, 17, 656-662. [CrossRef]

31. Sielatycki, J.A.; Chotai, S.; Stonko, D.; Wick, J.; Kay, H.; McGirt, M.J.; Devin, C.J. Is obesity associated with worse patient-reported outcomes following lumbar surgery for degenerative conditions? Eur. Spine J. 2016, 25, 1627-1633. [CrossRef]

32. Gaetani, P.; Aimar, E.; Panella, L.; Debernardi, A.; Tancioni, F.; Rodriguez y Baena, R. Surgery for herniated lumbar disc disease: Factors influencing outcome measures. An analysis of 403 cases. Funct. Neurol. 2004, 19, 43-49. [PubMed]

33. Asadi-Lari, M.; Tamburini, M.; Gray, D. Patients' needs, satisfaction, and health related quality of life: Towards a comprehensive model. Health Qual. Life Outcomes 2004, 2, 32. [CrossRef] [PubMed]

34. Urban-Baeza, A.; Zarate-Kalfopulos, B.; Romero-Vargas, S.; Obil-Chavarria, C.; Brenes-Rojas, L.; Reyes-Sanchez, A. Influence of depression symptoms on patient expectations and clinical outcomes in the surgical management of spinal stenosis. J. Neurosurg. Spine 2015, 22, 75-79. [CrossRef] [PubMed]

35. Sinikallio, S.; Aalto, T.; Airaksinen, O.; Herno, A.; Kroger, H.; Savolainen, S.; Turunen, V.; Viinamaki, H. Lumbar spinal stenosis patients are satisfied with short-term results of surgery-younger age, symptom severity, disability and depression decrease satisfaction. Disabil. Rehabil. 2007, 29, 537-544. [CrossRef]

36. Schroeder, G.D.; Coric, D.; Kim, H.J.; Albert, T.J.; Radcliff, K.E. Are patient-reported outcomes predictive of patient satisfaction 5 years after anterior cervical spine surgery? Spine J. 2017, 17, 943-952. [CrossRef]

37. Copay, A.G.; Martin, M.M.; Subach, B.R.; Carreon, L.Y.; Glassman, S.D.; Schuler, T.C.; Berven, S. Assessment of spine surgery outcomes: Inconsistency of change amongst outcome measurements. Spine J. 2010, 10, 291-296. [CrossRef]

38. Chotai, S.; Sivaganesan, A.; Parker, S.L.; McGirt, M.J.; Devin, C.J. Patient-Specific Factors Associated With Dissatisfaction After Elective Surgery for Degenerative Spine Diseases. Neurosurgery 2015, 77, 157-163. [CrossRef]

39. Rief, W.; Shedden-Mora, M.C.; Laferton, J.A.; Auer, C.; Petrie, K.J.; Salzmann, S.; Schedlowski, M.; Moosdorf, R. Preoperative optimization of patient expectations improves long-term outcome in heart surgery patients: Results of the randomized controlled PSY-HEART trial. BMC Med. 2017, 15, 4. [CrossRef]

40. Schwarz, K.A.; Pfister, R.; Buchel, C. Rethinking Explicit Expectations: Connecting Placebos, Social Cognition, and Contextual Perception. Trends Cogn. Sci. 2016, 20, 469-480. [CrossRef]

41. Den Ouden, H.; Vos, R.C.; Rutten, G. Effectiveness of shared goal setting and decision making to achieve treatment targets in type 2 diabetes patients: A cluster-randomized trial (OPTIMAL). Health Expect. 2017, 20 , 1172-1180. [CrossRef]

42. Wei, H.; Zhou, L.; Zhang, H.; Chen, J.; Lu, X.; Hu, L. The Influence of Expectation on Nondeceptive Placebo and Nocebo Effects. Pain Res. Manag. 2018, 2018, 8459429. [CrossRef] [PubMed]

Publisher's Note: MDPI stays neutral with regard to jurisdictional claims in published maps and institutional affiliations. 